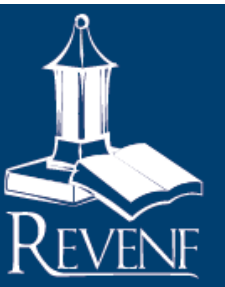

\title{
La realidad detrás de las farmacéuticas: sendas de una posmodernidad que no perdona ${ }^{1}$
}

\author{
Allan Rodríguez Artavia ${ }^{2}$
}

Institución: Hospital San Vicente de Paúl

\section{CÓMO CITAR}

Rodríguez, A. (Abril, 2014) La realidad detrás de las farmacéuticas: sendas de una posmodernidad que no perdona. Rev. Enfermería Actual de Costa Rica, 26, 1-13. Recuperado de <http://www.revenf.ucr.ac.cr/farmaceutica.pdf> ISSN 1409-4568

\section{RESUMEN}

El siguiente artículo se origina de la necesidad actual de analizar cuál es la realidad en torno a la industria farmacéutica a partir de los postulados de la (post) modernidad. Para su formulación, se efectuó una revisión bibliográfica en las bases de datos de la Biblioteca Virtual de Salud (Bireme) y en la Scientific Electronic Library Online (SciELO), en donde tras la aplicación de criterios de inclusión determinados, se obtuvo un total de 23 artículos que fungieron como base del planteamiento hecho. En cuanto a los principales resultados, en trece de los documentos analizados se critica el proceso de comercialización que ha dictado la pauta por seguir en la producción farmacéutica; luego, en otros siete se plantea que en dicha industria se restringe el acceso a los medicamentos en los países o regiones con menor poder adquisitivo, dado que existe resistencia a financiar investigaciones referentes a enfermedades que no son lucrativas. Como conclusión se afirma que en la actualidad, debido a los procesos propios de las políticas neoliberales y al capitalismo que envuelven a la producción farmacéutica, se perdió como objetivo la salud de la comunidad, trasformando dicho derecho en un proceso de marketing y mercantilismo.

Palabras clave: farmacéutica, medicalización, medicamento, modernidad, posmodernidad.

\footnotetext{
${ }^{1}$ Fecha de recepción: 25 de agosto del 2013

Fecha de aceptación: 15 de diciembre del 2013

${ }^{2}$ Enfermero Obstetra. Hospital San Vicente de Paul. Profesor de la maestría en Enfermería ginecológica, obstétrica y perinatal de la

Universidad de Costa Rica. Correo electrónico: allanrodriguez.a@gmail.com
} 


\section{The reality behind the pharmaceutical industry, postmodernity with no mercy ${ }^{1}$}

Allan Rodríguez Artavia ${ }^{2}$

Institution: San Vicente de Paul Hospital

CITED

The following article analyzes the current need in the light of the principles of the (post) modernity about the pharmaceutical industry. For its formulation, we conducted a literature review in the databases of the Virtual Health Library (BIREME), and the Scientific Electronic Library Online (SciELO), where after applying predetermined inclusion criteria, it was possible to obtain a total of 23 items that served as the analysis base. Between the main results obtained in thirteen of the documents analyzed it criticizing the marketing process that has set as standard to follow in pharmaceutical production. In seven of the articles it is argued that by in pharmacological industry, there is limited access to medicines in countries or regions with less purchasing power, as there is reluctance to fund research related to diseases that are not profitable. It conclude that given currently own processes of neoliberal policies and capitalism that surround pharmaceutical production, lost focus on the health of the community, transforming such right in a process of marketing and commercialism.

Keywords: medicalization, medicines, modernity, pharmaceutical, postmodernism.

\footnotetext{
${ }^{1}$ Date of reception: august 25, 2013

Date of acceptance: december 15,2013

${ }^{2}$ Nurse Obstetrician. San Vicente de Paul Hospital. Professor of gynecological, obstetric and perinatal nursing from the University of

Costa Rica. E mail: allarodriguez.a@gmail.com
} 


\section{INTRODUCCIÓN}

En las últimas décadas es evidente el avance en el desarrollo de nuevas tecnologías, cuyo fin es mejorar la condición de salud al crear tratamientos pensados para enfrentar problemas de salud que agobiaban a grupos dentro de las comunidades: dicho cambio funcionó como un feedback para la investigación de nuevos tratamientos.

Durante la modernidad, es palpable que la producción de estas tecnologías ha pasado a manos de grandes empresas más interesadas en los beneficios económicos que en la mejora de los padecimientos en grupos vulnerables. Contrario a la situación anterior, el concepto de modernidad, para Lyon (2000) es un "orden social que surgió tras la ilustración (...), y que está relacionado con la fe en el progreso y el poder de la razón humana para promover la libertad" (pp. 47-48), definición que orienta mejor respecto de cuestiones de ubicación y características del concepto. Dicho término, como lo menciona Habermas (1989), citado por Wallace (sf), "ha expresado una y otra vez la ciencia de una época que se mira a sí misma en relación con el pasado, considerándose resultado de una transición desde lo viejo hacia lo nuevo" (p.1), fenómeno que ha provocado la suma de ideologías liberales y capitalistas que pretenden, como lo menciona Adorno y Horkheimer (1988),

presentar al consumidor todas las necesidades como si pudiesen ser satisfechas por la industria cultural, pero también organizar esas necesidades en forma tal que el consumidor aprenda a través de ellas que es sólo y siempre un eterno consumidor, un objeto de la industria cultural (p.12).

La situación mencionada no es indiferente a la industria farmacéutica, la cual, partiendo de esa aculturación capitalista y neoliberal, ha ideado estrategias y acciones que incitan a la "promoción y la comercialización de los medicamentos en el contexto de una lógica de mercado dominante, orientada principalmente al propósito de ampliar la oferta y la demanda" (Cabral, 2008, p.586), razón por la que se afirma que ya no se busca la mejora en una cierta condición de salud de un usuario, por el contrario, en el presente la casa farmacéutica produce medicamentos para su consumo indiscriminado y para situaciones que antes no eran consideradas problemas de salud, al punto de que ha caído en los juegos y premisas de una cultura industrial ( Adorno y Horkheimer) que establece, a partir de las tendencias del capitalismo, un marketing, y la acumulación de capital, como objeto primario de su accionar.

En la actualidad, dado los efectos de esta tendencia, se presenta una realidad distorsionada, donde por una parte se muestra un gran grupo de la población mundial que carece de productos necesarios y, por otro lado, numerosas poblaciones consumen medicamentos de forma excesiva ante las nuevas problemáticas "inventadas" en salud.

Dada la situación anterior, y considerando la metodología de revisión bibliográfica, surge la idea de indagar acerca de las consideraciones de los distintos autores en relación con las implicaciones de las tendencias 
de la modernidad en la industria farmacéutica, con el fin de conocer y de valorar la afectación interna de esta industria (cuyo fin, en un inicio es curar la enfermedad), y el servicio que brinda a la comunidad.

Esta investigación corresponde a una revisión bibliográfica en la que se recurrió a las bases de datos Biblioteca Virtual de Salud (Bireme) y Scientific Electronic Library Online (Scielo) para localizar documentos bibliográficos analizados, , detectados con el uso de términos descriptores ("medicalización", "farmacéuticas and enriquecimiento", "medicamentos and lucro", "patentes farmacéuticas" "medicamentos and capitalismo" y "negocio farmacéutico") y de la búsqueda con operadores lógicos booleanos, (and, or, not); al final, se obtuvo 84 artículos que cumplían los criterios de la investigación.

Luego, mediante un proceso de depuración de los datos hallados, bajo la metodología de lectura crítica, se seleccionó un total de 23 artículos que cumplían con los criterios de inclusión. Cabe mencionar que dentro de la búsqueda se utilizaron los mismos descriptores en el buscador en línea Google Académico, como estrategia ampliada para el recabo de información.

De esta manera y por la gran cantidad de resultados, se limitó la búsqueda considerando los siguientes aspectos: Que los documentos se encuentren publicados en revistas indexadas y en formato de artículo científico; que puedan ser consultados de manera completa; la temática tratada corresponda al área de la venta o expansión farmacéutica; la publicación no sea mayor a los diez años.

Posterior a la lectura y análisis de los documentos que fueron seleccionados tras la búsqueda electrónica, se realizó una síntesis de cada uno, así como de los principales resultados y conclusiones. Posteriormente, se realizó un recuento de las coincidencias, diferencias y argumentos que se presentan en relación con cada uno de ellos.

\section{Consideraciones éticas}

Para cada material usado, se respetó los derechos de autor y, a través del texto, se incorporó las citas bibliográficas correspondientes.

\section{DESARROLLO}

En torno al tema, Alcántara (2012) establece que el sector salud en la actualidad se encuentra influenciado por el modelo hospitalocéntrico, mecanicista, individualista y curativista de los Estados Unidos, el cual se ha extendido a muchas sociedades en vías de desarrollo, hecho que ha pasado a ser un fenómeno de transculturación, con dependencia a los medicamentos y un obstáculo para solucionar los problemas de salud en estas naciones.

Aunado a lo anterior, en trece de los documentos analizados (Ahmad y cols, 2011; Borrell y Merino, 2006; Vara, 2008; Molina y Rivas, 2003; Macías, 2010; Milward, 2008; Carvajal, 2009; Cabral, 2008; Torres, 2010; Ugalde y Hómedes , 2009; Martínez, 2003; Cuello de Oro y López, 2007 y Faraone y cols 2009) se critica el 
proceso de comercialización imperante en la industria farmacéutica que la ha convertido en un "buen negocio", como lo menciona Carvajal (2009).

En relación con esta misma temática, Macías (2010) plantea la preocupación de que el mercado farmacéutico de las vacunas se encuentre apuñado por numerosas empresas, lo cual significa poca participación de entidades e instituciones nacionales e internacionales en tales procesos, situación que desemboca en que las corporaciones multinacionales sean las que asuman el diseño, desarrollo, producción y comercialización de las vacunas (Macías, 2010), sin considerar las necesidades reales de las comunidades en el mundo.

Todo lo mencionado aparece en tres artículos donde se critica la estrategia comercial del uso de las patentes por parte de las farmacéuticas con el fin de establecer una especie de oligopolio o monopolio en el cual el precio ya no es fijado en el encuentro entre la oferta y la demanda, sino por la firma (Carvajal, 2009), al punto de que prevalece un "derecho al lucro" de las multinacionales por encima del derecho fundamental a la vida (Torres, 2010). Al respecto, Torres (2010) considera que la salud ingresó a la esfera económico-mercantil en la medida en que se ha convertido en un objeto de consumo, en un producto que puede ser fabricado por los laboratorios farmacéuticos y consumido por los enfermos reales o posibles, cambio que ha favorecido que el nuevo modelo empresarial de la Big Pharma priorice las ventas y la dependencia a los medicamentos, estableciendo como eslogan "el fin principal de la promoción no es informar, sino persuadir", sentencia que se visualiza cuando transforman conductas inusuales en problemas de salud (Ugalde y Hómedes 2009).

Por otra parte, en cinco de los artículos analizados (Nwobike, 2005; Orta, 2008; Cabral 2008 y Torres, 2010), se plantea que en los países o regiones con menor poder adquisitivo, o en vías de desarrollo, las farmacéuticas limitan el acceso a los medicamentos, además de que han olvidado la formulación de medicaciones para los males o enfermedades comunes, al punto de que lo que impera en el mercado comercial son las “enfermedades creadas o inventadas". En el artículo de Nwobike (2005), se menciona que la única participación de los países más desfavorecidos en cuanto a adquirir medicamentos surge cuando sus ciudadanos se involucran en pruebas experimentales para aprobar un protocolo de investigación para un nuevo tratamiento; de igual forma, el autor expone el actuar incorrecto de las farmacéuticas en cuanto al acceso a medicamentos, como por ejemplo para la pandemia de VIH/SIDA, debido a resistencia en cuanto a financiar investigaciones acerca de enfermedades no lucrativas.

Por otro lado, en cuanto a la medicalización de la vida cotidiana, en cinco de los documentos analizados, (Rodríguez, 2008; Faraone y cols, 2010; Cabral, 2008; Rodríguez, 2010 y Ortún 2004) se cuestiona el actuar de la medicina y farmacia, puesto que dichas ciencias ya no se ocupan de combatir la enfermedad, sino implantar una determinada manera a la sociedad, de acuerdo con sus intereses. Al respecto, los autores concluyen que la línea divisoria entre el "sano" y el "enfermo", es más amplia, lo cual ensancha el alcance de la definición de determinadas patologías dentro de las que se incluye un número mayor de enfermos hasta, por ende, medicalizar los cuerpos. 
Otra situación que acontece en la actualidad es que en los hogares, para nada extraña, es que es muy común encontrar gran cantidad de medicamentos no prescritos, los cuales se almacenan en lugares poco seguros, hecho que implica mayor riesgo de automedicación, y medicalización (Tourinho y cols, 2008).

Siempre en relación con el tema, Vara (2008) muestra que los médicos de cierta parte de la comunidad médica, de un país en particular, establecen una gran interacción con la industria farmacéutica; aparte, informa respecto de que este cuerpo profesional considera "apropiado" recibir productos farmacéuticos, aunque reconozca que estas actividades de promoción "influyen en la prescripción y elevan el costo final de los productos", cuyo perjudicado es el usuario. De hecho, en cuatro de los documentos examinados (Rodríguez, 2008; Faraone y cols, 2010, Palma y Vilaça, 2012 y Ugalde y Hómenes, 2009) se evidencia que las farmacéuticas han creado nuevas estrategias de marketing y difusión, con el fin penetrar no solo en el grupo médico, sino dirigir las acciones de los sujetos no médicos, particularmente maestros y asociaciones de padres.

Dado lo anterior, Rodríguez (2010) concluye que, particularmente desde el campo de las ciencias de la comunicación, preponderantes imprescindible problematizar la ingeniería simbólica y la estrategia discursiva puesta en marcha por la industria farmacéutica, puesto que, a través de sus estrategias de marketing, el mercado continúa expandiéndose y olvidando en parte su fin constitutivo.

Aún cuando un número de empresas farmacéuticas rinden cuentas de su ciudadanía corporativa o de sus actividades de responsabilidad empresarial, pocas incluyen en sus declaraciones su misión de derechos humanos, en general o de derecho de la salud en particular; incluso, menos parecen haber examinado cuidadosamente sus políticas a través del lente del derecho al disfrute del más alto nivel posible de salud (Hunt y Khosla, 2008) .

Tal como fue abordado por Jameson (1991), en los tiempos actuales se experimenta "la forma más pura de capitalismo de cuantas han existido, experimentando una ampliación prodigiosa del capital hasta en territorios antes no mercantilizados" (p.81). Este pensamiento concuerda con la conclusión de otros autores (Rodríguez, 2008; Faraone y cols, 2010; Palma y Vilaça, 2012; Ugalde y Hómenes, 2009), quienes opinan que por esta propensión de adquirir más dinero, las empresas farmacéuticas han elaborado distintas estrategias de marketing dirigidas a personas que no prestan una atención directa en salud (maestros, padres, $u$ otros), de modo que no influyen de manera directa en la toma de decisiones para la compra de los medicamentos, mas continúan ampliando el mercado del consumo a favor del capitalismo.

Respecto de la automedicación y el aumento de medicamentos en los hogares, de Tourinho y cols (2008), concuerdan con el pensamiento de Marcuse (1991), al mencionar que "una sociedad que cada día parece más capaz de satisfacer las necesidades de los individuos por medio de la forma en la que está organizada, priva la independencia de pensamiento" (pp. 31-32). Lo anterior se refleja en la falsa expectativa respecto de la libertad de escoger un producto o medicamento para remediar un mal o dolencia, sin tener la consciencia de que esta "supuesta libertad de elección" no escapa a la oferta y stock ofrecido por las farmacéuticas y supermercados, 
donde ya de antemano han establecido cual es la necesidad de consumo bajo el modelo impuesto por la cultura capitalista.

La percepción del autoconsumo y auto prescripción de medicamentos debe de mirarse con cuidado, dado que, como lo acota Castro-Orellana (2009), esta tendencia ha surgido como una política de neoliberalismo y de la industria farmacéutica en la cual

el individuo debería gestionar sus propios riesgos, articular toda una forma de vida en la cual resulta preciso invertir en el propio cuidado y la protección de uno mismo. Así, se abre un nuevo horizonte de necesidades y demandas que satisfacer, un territorio de amenazas rentables en donde ingresan las ofertas de consumo de la industria de los seguros y de la industria de la salud (pp. 21-22).

Aunado a lo anterior, Soriano (2002) añade: "Poca industria farmacéutica nos queda ya de aquella cuyo objetivo es simplemente fabricar medicamentos, obviando la finalidad empresarial" (p. 82), afirmación acorde a las conclusiones expuestas por Rodríguez (2010), dentro del resultado de esta revisión, dado que por las situaciones de mercado y la influencia del capitalismo, se ha ido perdiendo la idea de la preservación de la salud y la producción en serie de medicamentos se ha convertido en toda una industria comercial que vive de las ventas. Acerca del tema, dentro de su planteamiento. Goodwin (2007) se cuestiona:

¿Es excesiva la influencia de la industria farmacéutica sobre la prescripción de los medicamentos que realizan los médicos? Sí. En el momento presente, los gastos que dedican las compañías farmacéuticas a la Formación Médica Continuada, son mayores a los que dedican a cualquier otro aspecto de su actividad (p. 25).

La respuesta de Goodwin concuerda con las conclusiones planteadas por Vara (2008), quien atribuye a la industria farmacéutica una interacción constante con el gremio médico, realidad que influye en la prescripción de los medicamentos, y en el costo final pagado por el consumidor. Del mismo modo piensa Castro-Orellana (2009), quien comenta:

De hecho, la industria farmacéutica se rige por un criterio que no consiste en la extinción de la enfermedad o la minimización de las incertidumbres relacionadas con la salud, sino más bien por una lógica de preservación y administración multiplicadora de las dolencias y las inquietudes (p.22).

Dicho planteamiento establece una correlación con lo expuesto por Ugalde y Hómedes (2009), quienes reflexionan en cómo las farmacéuticas, valiéndose de todo tipo de estrategia, transforman conductas inusuales en problemas de salud. A su vez, ambos autores analizan el objetivo de la producción de las farmacéuticas: 
Al poder farmacéutico le interesa particularmente una cosa: la existencia de sujetos que posean conductas de consumo farmacológico cada vez más intensas. Esto quiere decir que las farmacéuticas trabajan para aquellos que pueden comprar un medicamento y no para quienes poseen una enfermedad. Por este motivo, el mercado de la salud ya no funciona en torno al problema enfermo/enfermedad, sino que gira alrededor de las relaciones que pueden establecerse entre el fármaco y el estilo de vida de los individuos (Castro, 2009, p.22).

Esta conclusión coincide con lo expuesto por Nwobike (2005), quien anota que, en muchos países, dentro de las prácticas de las empresas farmacéuticas se ha establecido limitantes para el acceso a los medicamentos, dado que estas empresas se resisten a financiar investigaciones asociadas a enfermedades que no son lucrativas, un claro reflejo de la pérdida del norte original de este tipo de industria.

En lo referente a la creación de oligopolios y monopolios por parte de las industrias farmacéuticas, Pérez (2004) coincide con Carvajal (2009), al afirmar que con el manejo de las patentes establecen una forma de regulación de los precios que no se centra en la relación de oferta/ demanda, sino a las disposiciones de la industria. Acerca de este mismo tema, Pérez (2004) añade que

las dos terceras partes del mercado mundial de medicamentos está en manos de 20 grandes monopolios farmacéuticos. Pero no solo se trata de una concentración financiera. En el caso de los Estados Unidos, el lobby farmacéutico en Washington, se ha hecho tan poderoso como el lobby judío y el de la industria militar alrededor del poder político (p.7).

La circunstancia mencionada ha originado que estas compañías financien a los partidos políticos, los cuales les brindan el apoyo necesario para impulsar políticas y consideraciones que benefician sus estrategias mercantiles y capitalistas.

Respecto de este fenómeno, Vasen (2008) apunta que,

la industria farmacéutica suele presentarse a sí tan bien intencionada como innovadora, como un sector que invierte grandes sumas en el desarrollo de nuevos productos que serán capaces de salvar muchas vidas, y ante cualquier intento de regular sus prácticas o sus ganancias, reaccionan resaltando lo que sucedería si no hubiera innovación en absoluto. La realidad muestra que este discurso no es cierto, la presencia de medicamentos en el mercado es muy importante y no responde a la innovación para salvar vidas sino a una innovación parcial (con una cuota de imitación) para ganar mercado (p.417).

Esta misma idea es respalda por las conclusiones encontradas y referidas por Torres (2010), dado que desde que la salud ingresó a la esfera del comercio y se ha convertido en objeto de consumo, la forma de pensar de esta industria ha cambiado: el bienestar de la población ya no es la prioridad, sino lo que se puede obtener económicamente de ella. 
En relación con la industria farmacéutica, Lorenzo y Garrafa (2011) anotan que

La industria farmacéutica multinacional es hoy en día uno de los más poderosos conglomerados económicos del planeta. El hecho de que las principales patentes pertenezcan a solo 15 empresas y que la mayoría de los ensayos clínicos estén relacionados con el control de enfermedades crónicas o estados premórbidos (tales como diabetes, asma, dislipidemias e hipertensión), crea un mercado típicamente cautivo, con estabilidad para el negocio y crecimiento lucrativo constante (p.167).

La opinión de Lorenzo y Garrafa es similar a lo expuesto por Hunt y Khosla (2008), quienes indican que de parte de muchas empresas del sector de los medicamentos se ha olvidado el enfoque de salud como derecho y han convertido la atención y fabricación de fármacos en un mero negocio.

Un claro ejemplo de este tipo de situaciones lo propone Fortune 500 (2013) en su Annual ranking of largest corporations 2012, en cuyo ranking se destaca, por su solidez y ganancias, empresas como Pfizer, ubicada en el puesto 126, con beneficios o ingresos por 67932 millones de dólares; Novartis en el puesto 157, con ingresos anuales de 59375 millones de dólares; Sanofi, en el puesto 201, con ingresos de 48747 millones de dólares anuales; GlaxoSmithKline, en el puesto 23,1 con ingresos de 43907 millones de dólares al año o Eli Lilly, en el puesto 454, con ingresos anuales de 24287 millones de dólares: todas son farmacéuticas reconocidas que se han convertido en verdaderas máquinas de producción de dinero, a costa de la población y la salud.

Asimismo, de acuerdo con las conclusiones planteadas por Macías (2010), en torno a la patentización de los medicamentos, como un fenómeno que encierra un ciclo donde las farmacéuticas asumen el diseño, desarrollo, producción y comercialización, convergen con lo expuesto por Melé (2006) quien anota en su trabajo que

parte de las críticas seguramente más conocidas a las compañías farmacéuticas es el precio excesivo de los medicamentos que impiden el acceso a los pobres a medicamentos cruciales para su vida (...).Como respuesta las compañías farmacéuticas defienden enérgicamente la necesidad de patentes que impidan a terceras compañías competir con fármacos genéricos a bajo precio durante muchos años. Es conocido el litigio de algunas compañías farmacéuticas contra el gobierno de África del Sur, por pretender fabricar e importar medicamentos genéricos para tratar a los enfermos de SIDA. Una empresa de la India que fabricaba los genéricos deberá dejar de hacerlo ya que el parlamento de la India lo ha prohibido, como reacción a presiones del sector farmacéutico y de organismos reguladores del comercio internacional (p.4).

Como se ha mencionado a largo de esta revisión, es evidente que, en búsqueda de más ganancias, este tipo de empresas han implementado diversas estrategias para garantizarse la comercialización única del producto, sin importar la salud como tal y precio final al consumidor. Se puede agregar que se ha perdido el objetivo principal por lo que la farmacia fue creada y su meta en la actualidad se basa en lo comercial únicamente. 
Como dato último a la reflexión planteada por Hunt y Rajat Khosla (2008) en torno a los derechos de las personas respecto del acceso a los medicamentos, la interpretación de Collado (2009) manifiesta la realidad actual en torno a lo que el capitalismo y las farmacéuticas pretenden: "En el sistema capitalista en el que estamos inmersos, el derecho a la salud se ha convertido en un bien privado al que únicamente tienen acceso aquellos que se lo pueden costear.." (sp), realidad que se enfrenta hoy en torno al derecho a la salud y al acceso a los medicamentos.

\section{CONCLUSIONES}

A través del planteamiento de los distintos autores, se conoce que la incorporación del modelo de desarrollo capitalista y neoliberal a la industria farmacéutica ha cambiado la forma en la que hace llegar los medicamentos que produce a la población.

En el presente, el objetivo de la industria no se aboca a producir medicamentos para asegurar la salud de la población, sino a crear estrategias de marketing y consumo para medicar desde edades más tempranas y ganar más dinero.

Las farmacéuticas han olvidado que existen regiones y países que carecen de medicamentos para atacar o contrarrestar los padecimientos de sus pobladores, los cuales, al no generar renta en su producción y consumo, son ignorados, dado que se prefiere priorizar las enfermedades y situaciones lucrativas.

Desde el actual sistema de producción en la industria farmacéutica, el derecho a la salud quedó relegado: solo se puede tener acceso a ese derecho si se cuenta con los medios económicos para poder financiarlo.

\section{BIBLIOGRAFÍA}

Adorno, T., Horkhaimer, M. (1988). La industria cultural. Iluminismo como mistificación de masas (Dialéctica del humanismo) Buenos Aires: Sudamericana.

Ahmad, M., Akhtar, N., Awan, M.H.A., Murtaza, G. (2011). Ethical Evaluation of Pharmaceutical Marketing in Pakistan. Revista Acta Bioethica, 17 (2), 215-224. Recuperado de http://www.redalyc.org/articulo.oa?id=55420909008

Alcántara, G. (2012). Transculturación del consumismo sanitario y medicalización en la globalización. Revista electrónica de Portales Médicos.com. Recuperado de

http://www.portalesmedicos.com/publicaciones/articles/4955/1/Transculturacion-del-consumismo-sanitario-ymedicalizacion-en-la-globalizacion.html

Borrell, J., Merino, A. (2006). Efectos perversos de la regulación farmacéutica en España: ¿hasta dónde se traslada la competencia? Gaceta Sanitaria, 20 (2),41-50. Recuperado de http://zl.elsevier.es/es/revista/gacetasanitaria-138/articulo/efectos-perversos-regulacion-farmaceutica-espana-13089482 
Cabral, J. (2008). Nuevas tendencias de la medicalización. Revista Ciência \& Saúde Coletiva, 13 (Sup), 579-587. Recuperado de http://www.scielo.br/pdf/csc/v13s0/a07v13s0.pdf

Carvajal, Y. (2009). Patentes farmacéuticas y acuerdos comerciales. Revista Cuadernos Médico Sociales. 49 (2) Recuperado de http://www.colegiomedico.cl/Portals/0/files/biblioteca/publicaciones/cuadernos/49_2.pdf

Castro-Orellana, R. (Enero-junio,2009). Capitalismo y medicina: los usos políticos de la salud. Revista Ciencia Politica, 7. Recuperado de http://www.revistas.unal.edu.co/index.php/cienciapol/article/view/16250

Collado, L. (2009). La mercantilización del derecho a la salud: patentes farmacéuticas. Anuario de acción humanitaria y derechos humanos, 6, 91-114. Recuperado de http://dialnet.unirioja.es/servlet/articulo?codigo $=3313955$

Cuello de Oro, D., López, C. (2007). Las patentes como mecanismo de apropiación de rentas económicas. Un estudio empírico en el sector farmacéutico español. Revista Europea de Dirección y Economía de la Empresa, 16 (1), 11-28. Recuperado de http://www.aedem-virtual.com/articulos/123679137600.pdf

Faraone, S., Barcala, A., Torricelli, F., Bianchi, E., Tamburrino, M. (junio, 2009). La industria farmacéutica en los procesos de medicalización/ medicamentalización en la infancia. Revista Margen 54. 54. Recuperado de http://www.margen.org/suscri/margen54/faraone.pdf

Faraone, S., Barcala, A., Torricelli, F., Bianchi, E., Tamburrino, M. (julio-setiembre, 2010). Discurso médico y estrategias de marketing de la industria farmacéutica en los procesos de medicación de la infancia en Argentina. Revista Interface: Comunicação Saúde Educação, 14 (34), 485-97 Recuperado de http://www.scielo.br/pdf/icse/v14n34/aop1110.pdf

Fortune 500 (julio, 2013). Annual ranking of largest corporations 2012. Recuperado de http://money.cnn.com/magazines/fortune/

Goodwin, G. (abril, 2007). Intereses encontrados y Honestidad. Revista World Phychiatry edición en español, 5 (1), 25-26 Recuperado de

http://www.wpanet.org/uploads/Publications/WPA_Journals/World_Psychiatry/Past_Issues/Spanish/wpa-042007-spa.pdf

Hunt, P., Khosla, R. (junio, 2008). El derecho humano a los medicamentos. Revista Internacional de Derechos Humanos, 5 (8). Sao Paulo. Recuperado de http://www.surjournal.org/esp/conteudos/getArtigo8.php?artigo=8,artigo_hunt.htm

Jameson, F. (1991). El posmodernismo o la lógica cultural del capitalismo avanzado. Buenos Aires: Paidós

Lorenzo, C; Garrafa, V. (2011). Ensayos clínicos, Estado y sociedad: ¿dónde termina la ciencia y empieza el negocio? [Debate]. Revista Salud Colectiva, 7 (2),166-170. Recuperado de http://www.scielo.org.ar/pdf/sc/v7n2/v7n2a08.pdf 
Lyon, D. (2000). Postmodernidad, Capt. 3: El malestar en la modernidad. Madrid: Alianza Editorial.

Macías, C. (enero-abril, 2010). Respuestas institucionales y corporativas a la pandemia de 2009 América Latina ante los actores multinacionales en la producción de medicamentos. Revista Desacatos, 32, 63-88. Recuperado de http://www.scielo.org.mx/pdf/desacatos/n32/n32a6.pdf

Marcuse, H. (1991). El hombre Unidimensional. Boston: Beacon Press.

Martínez, M. (2003). Las patentes en la industria farmacéutica: entre la ética y los derechos de propiedad. Revista salud pública. 5 (1): 18-23. Recuperado de http://www.scielo.org.co/pdf/rsap/v5n1/v5n1a02.pdf

Melé, D. (2006). Las cinco mayores compañias farmacéuticas: imagen corporativa y críticas en responsabilidad social. Cátedra economía y ética. IESE Bussines School- Universidad de Navarra. Recuperado de http://www.cp.org.ar/imagenes/columnistas/archivos/merecen\%20las\%20farmaceuticas\%20la\%20imagen\%20que $\% 20$ se $\% 20$ tienen $\% 20$ de $\% 20$ ellas\%20mele.pdf

Milward, C. (julio, 2008). Patentes farmacêuticas e saúde pública: desafíos à política brasileira de acesso ao tratamento anti-retroviral. Revista Cad. Saúde Pública, Río de Janeiro, 24 (7), 1467-1478. Recuperado de http://www.scielo.br/pdf/csp/v24n7/02.pdf

Molina, R., Rivas, J. (julio-setiembre, 2003). Sobreprecio y acceso a los medicamentos: el caso de los medicamentos esenciales en México. Revista Cad. Saúde Pública, 14 (3), 501-506. Recuperado de http://www.scielosp.org/pdf/csp/v14n3/0086.pdf

Nwobike, J. (2005). Empresas farmacéuticas y acceso a medicamentos en los países en desarrollo: el camino a seguir. Revista Internacional de Derechos Humanos, 2 (3). Recuperado de http://www.scielo.br/pdf/sur/v3n4/es_07.pdf

Orta, I. (octubre-diciembre, 2008). Ciencia y política: el acceso a los medicamentos en el mundo de hoy. Revista Habana Ciencia Médica, Vol. VII. (4). Recuperado de http://scielo.sld.cu/pdf/rhcm/v7n4/rhcm12408.pdf

Ortún, V. (2004). Patentes, regulación de precios e innovación en la industria farmacéutica. Cuadernos económicos del Centro de Investigación en Economía y Salud (I.C.E), 67. Recuperado de http://www.revistasice.info/cachepdf/CICE_67_191-207_CA88FD0653698542FF0182979B745287.pdf

Palma, A., Vilaça, M. (julio-setiembre, 2012). Conflitos de interesse na pesquisa, produção e divulgação de medicamentos. Revista História, Ciências, Saúde, 19 (3), 919-932. Recuperado de http://www.scielo.br/pdf/hcsm/v19n3/08.pdf

Pérez, J. (setiembre-diciembre, 2004). Dos enfoques sobre los medicamentos y la industria farmacéutica. Revista Cubana Salud Pública, 30 (4). Recuperado de http://bvs.sld.cu/revistas/spu/vol30_4_04/spu07404.htm 
Rodríguez, S. (2008). El proceso de medicalización y sus consecuencias. Entre la moral, el poder y el negocio. Intersticios. Revista sociológica de pensamiento crítico, 2 (2). Recuperado de http://www.intersticios.es/article/view/2714/2128

Rodríguez, P. (julio, 2010). La medicalización como estrategia biopolítica. A parte Rei. Revista de Filosofia, 70. Recuperado de http://serbal.pntic.mec.es/ cmunoz11/zoya70.pdf

Soriano, J. (noviembre, 2002). Bonificaciones, descuentos y rápeles. Revista Offarm, 21(10) 82-83. Recuperado de:

http://apps.elsevier.es/watermark/ctl_servlet? $\mathrm{f}=10 \&$ pident_articulo $=13039712 \&$ pident_usuario $=0 \& p i d e n t$ revist $\mathrm{a}=4 \&$ fichero=4v21n10a13039712pdf001.pdf\&ty=107\&accion=L\&origen=doymafarma\&web=www.doymafarma .com\&lan $=$ es

Torres, A. (2010). Medicamentos y transnacionales farmacéuticas: impacto en el acceso a los medicamentos para los países subdesarrollados. Revista Cubana de Farmacia, 45(1), 97-110. Recuperado de http://scielo.sld.cu/pdf/far/v44n1/far12110.pdf

Tourinho, F., Bucaretchi, F., Stephan, C., Cordeiro, R. (2008). Home medicine chests and their relationship with self-medication in children and adolescents. Revista Journal de Pediatría, 84 (5). Recuperado de http://www.scielo.br/pdf/jped/v84n5/v84n5a07.pdf

Ugalde, A., Hómedes, H. (2009). Medicamentos para lucrar. La transformación de la industria farmacéutica. Salud Colectiva, 5(3), 305-322. Recuperado de http://www.scielo.org.ar/pdf/sc/v5n3/v5n3a02.pdf

Vara, A. (2008). Cómo medicalizar la vida diaria: la creación de enfermedades o "disease mongering". Revista EVIDENCIA - Actualización en la Práctica Ambulatoria - Septiembre/Octubre. Recuperado de http://www.foroaps.org/files/ygjytgkgyt.pdf

Vasen, F. (2008) Regulación tecnológica y valores sociales: un análisis del caso farmacéutico. Revista Scientle Studia, 6 (3), 409-26. Sao Paolo. Recuperado de: http://www.scielo.br/pdf/ss/v6n3/v6n3a09.pdf

Wallace, D. (sf). Modernidad: un proyecto incompleto de Jurgen Habermas. Material del curso Coloquio de Investigación 1. Doctorado en Estudios de la Sociedad y Cultura, 2013. Universidad de Costa Rica. Prof. Dra. Vanessa Fonseca. 